\title{
Harnessing Buckling to Design Tunable Locally Resonant Acoustic Metamaterials
}

\section{Citation}

Wang, Pai, Filippo Casadei, Sicong Shan, James C. Weaver, and Katia Bertoldi. 2014. "Harnessing Buckling to Design Tunable Locally Resonant Acoustic Metamaterials." Physical Review Letters 113 (1). https://doi.org/10.1103/physrevlett.113.014301.

\section{Permanent link}

http://nrs.harvard.edu/urn-3:HUL.InstRepos:41461276

\section{Terms of Use}

This article was downloaded from Harvard University's DASH repository, and is made available under the terms and conditions applicable to Other Posted Material, as set forth at http:// nrs.harvard.edu/urn-3:HUL.InstRepos:dash.current.terms-of-use\#LAA

\section{Share Your Story}

The Harvard community has made this article openly available.

Please share how this access benefits you. Submit a story.

Accessibility 


\title{
Harnessing Buckling to Design Tunable Locally Resonant Acoustic Metamaterials
}

\author{
Pai Wang, ${ }^{1}$ Filippo Casadei, ${ }^{1}$ Sicong Shan, ${ }^{1}$ James C. Weaver, ${ }^{2}$ and Katia Bertoldi ${ }^{1,3, *}$ \\ ${ }^{1}$ School of Engineering and Applied Sciences, Harvard University, Cambridge, Massachusetts 02138, USA \\ ${ }^{2}$ Wyss Institute for Biologically Inspired Engineering, Harvard University, Cambridge, Massachusetts 02138, USA \\ ${ }^{3}$ Kavli Institute, Harvard University, Cambridge, Massachusetts 02138, USA
}

(Received 28 March 2014; published 3 July 2014)

\begin{abstract}
We report a new class of tunable and switchable acoustic metamaterials comprising resonating units dispersed into an elastic matrix. Each resonator consists of a metallic core connected to the elastomeric matrix through elastic beams, whose buckling is intentionally exploited as a novel and effective approach to control the propagation of elastic waves. We first use numerical analysis to show the evolution of the locally resonant band gap, fully accounting for the effect of nonlinear pre-deformation. Then, we experimentally measure the transmission of vibrations as a function of the applied loading in a finite-size sample and find excellent agreement with our numerical predictions. The proposed concept expands the ability of existing acoustic metamaterials by enabling tunability over a wide range of frequencies. Furthermore, we demonstrate that in our system the deformation can be exploited to turn on or off the band gap, opening avenues for the design of adaptive switches.
\end{abstract}

DOI: 10.1103/PhysRevLett.113.014301

PACS numbers: 46.32.+x, 46.40.Cd, 46.40.Ff

Artificially structured composite materials that enable manipulation and control of elastic waves have received significant interest in recent years [1], not only because of their rich physics, but also for their broad range of applications, including wave guiding [2-7], cloaking [8] and noise reduction [9-11]. An important characteristic of these heterogeneous systems is their ability to tailor the propagation of elastic waves due to the existence of band gaps-frequency ranges of strong wave attenuation. In phononic crystals, band gaps are generated by Bragg scattering [12], whereas in acoustic metamaterials, localized resonance within the medium is exploited to attenuate the propagation of waves. Analogous to the case of photonic crystals where split-ring resonators have been embedded within a medium to excite electromagnetic resonances [13,14], in acoustic metamaterials the internal resonance is typically realized by dispersing heavy inclusions coated with a soft layer into a matrix [15]. Interestingly, acoustic metamaterials are capable of manipulating waves with wavelengths much larger than the structural features of the system [15] and have been successfully exploited for vibration control [16,17], imaging [18], design of exotic elastic solids [19,20], and thermal management [21].

Most of the phononic and acoustic metamaterial configurations proposed to date are characterized by a passive response and operate at fixed frequency ranges, limiting the number of possible applications. In an effort to design tunable systems, it has been shown that Bragg-type band gaps can be controlled by instability-induced pattern transformations [22-24]. On the other hand, for acoustic metamaterials, tuning of functionalities has been achieved by altering the resonant frequency via piezoshunting [25], adaptive connectivity [26], and fluid-structure interactions [27].

Here, we report a new class of adaptive acoustic metamaterials whose response is controlled by mechanical deformation. In the proposed metamaterial, the elastomeric coating that typically surrounds the resonating mass is replaced by easy-to-buckle elastic beams, as shown in Fig. 1(a). When the system is compressed statically, these beams buckle at a relatively low level of applied uniaxial
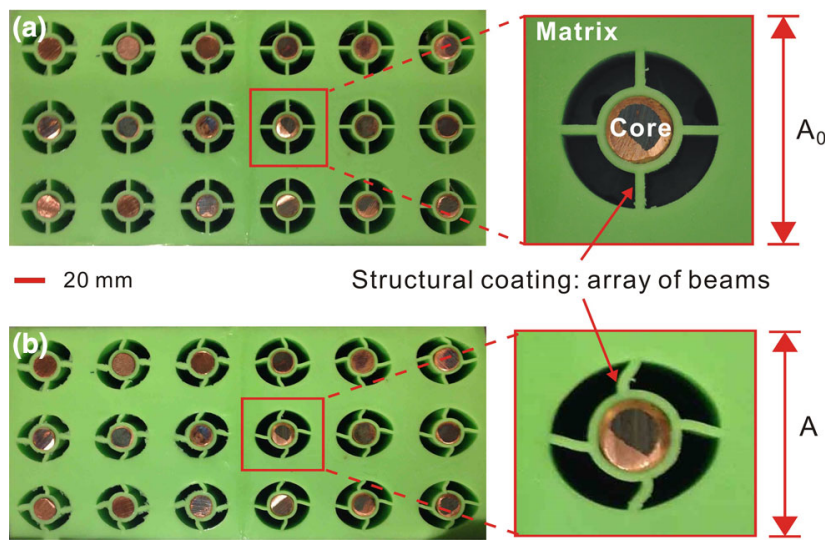

FIG. 1 (color online). Tunable acoustic metamaterial: (a) The undeformed configuration comprises resonating units dispersed into an elastomeric matrix. Each resonator consists of a metallic mass connected to the matrix through elastic beams, which form a structural coating. The black regions in the picture indicate voids in the structure. The unit cell size is $A_{0}=50.0 \mathrm{~mm}$. (b) When a compressive strain $\varepsilon=-0.10$ is applied in the vertical direction, buckling of the beams significantly alters the effective stiffness of the structural coating, which in turn changes the band gap frequency. 
strain $\varepsilon$, as seen in Fig. 1(b) for $\varepsilon=-0.1$. Interestingly, buckling dramatically alters the stiffness of the beams and consequently the natural frequency of the resonating units, which in turn determines the frequency range of the band gap.

Through a combination of analyses and experiments, we demonstrate that elastic instability and deformation can be exploited to effectively tune and even completely suppress the band gaps of the system. Hence, the metamaterial can be used as an acoustic switch that provides on or off capabilities.

To fully understand the effect of deformation on the propagation of small amplitude elastic waves in the proposed acoustic metamaterial, we focus on the elastic system shown in Fig. 1 (the structure is uniform in the outof-plane dimension with height of $50.0 \mathrm{~mm}$ ). Here we consider a square array of locally resonant unit cells with center-to-center distance $A_{0}=50.0 \mathrm{~mm}$, but the response of the system is not affected by the spatial arrangement of the resonators [28,29]. Each resonating unit comprises (i) a portion of the elastomeric matrix, (ii) a metallic circular core with radius $R_{0}=7.9 \mathrm{~mm}$, and (iii) four beams with length $L_{0}=16.9 \mathrm{~mm}$ and thickness $t_{0}=1.8 \mathrm{~mm}$ that connect the metallic core to the matrix.

Both the beams and the matrix are made of silicon rubber (Elite Double 32, Zhermack) with measured shear modulus $\mu_{0}=4.44 \times 10^{5} \mathrm{~N} / \mathrm{m}^{2}$, Poisson's ratio $\nu_{0}=0.499$, and density $\rho_{0}=1050 \mathrm{~kg} / \mathrm{m}^{3}$ (so that in the undeformed homogeneous material the elastic wave speeds are $c_{T}=$ $20.6 \mathrm{~m} / \mathrm{s}$ and $c_{L}=460.1 \mathrm{~m} / \mathrm{s}$ for shear and pressure waves, respectively). Differently, the metallic cores are built using copper rods (with shear modulus $\mu_{\mathrm{Cu}}=$ $4.78 \times 10^{10} \mathrm{~N} / \mathrm{m}^{2}$, Poisson's ratio $\nu_{\mathrm{Cu}}=0.34$ and density $\left.\rho_{\mathrm{Cu}}=8960 \mathrm{~kg} / \mathrm{m}^{3}\right)$.

We first investigate the effect of uniaxial compression on the static and dynamic responses of the system via finite element (FE) simulations using the commercial software ABAQUS/STANDARD. In the simulations we focus on a representative volume element comprising a single resonating unit [see Fig. 2(a)] and use periodic and Bloch-type boundary conditions for the static and dynamic analyses [23], respectively. Two-dimensional (2D) models are constructed using quadrilateral plane strain hybrid quadratic elements (Abaqus element type CPE8 H) and the response of the elastomeric matrix is described by a nearly incompressible Neo-Hookean model with initial shear modulus $\mu_{0}$ and Poisson's ratio $\nu_{0}$.

To begin with, we focus on the static response of the unit cell and perform a linear perturbation analysis to investigate the stability of the beams. A local instability is detected at $\varepsilon_{c r}=-0.0247$, associated with buckling of the two vertical beams. The postbuckling response of the system is then simulated by introducing small random imperfections into the initial geometry and performing a nonlinear static analysis. Snapshots of the deformed configurations at
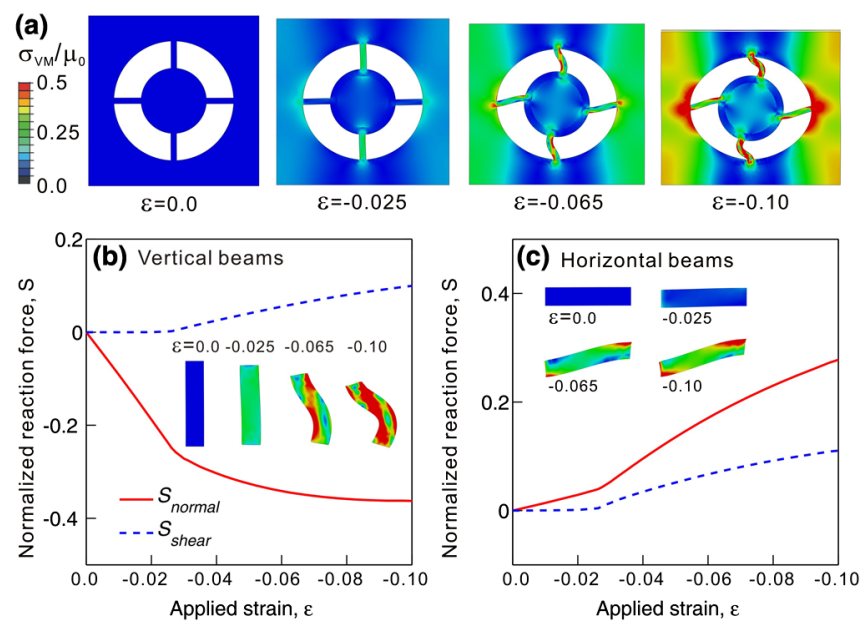

FIG. 2 (color online). Static response: (a) Distribution of the normalized Von Mises stress, $\sigma_{\mathrm{VM}} / \mu_{0}$ at different levels of applied strain $\varepsilon$. (b) Effect of the applied deformation on the reaction force transmitted by the vertical beams to the matrix. The normalized reaction forces acting both in axial (normal component), $S_{\text {normal }}=R_{\text {normal }} /\left(t_{0} \mu_{0}\right)$, and tangential (shear component), $S_{\text {shear }}=R_{\text {shear }} /\left(t_{0} \mu_{0}\right)$, are reported, $R_{\text {normal }}$ and $R_{\text {shear }}$ denoting the total reaction force measured at the end of the beam in the normal and tangential directions, respectively. (c) Effect of the applied deformation on the force transmitted by the horizontal beams to the matrix.

different levels of the applied strain are shown in Fig. 2(a). As predicted by the stability analysis, when the structure is compressed uniaxially in the vertical direction, the two vertical beams buckle at $\varepsilon_{c r}$. Moreover, the buckling of vertical beams is also accompanied by rotation of the metallic core that, in turn, results in the stretching of the two horizontal beams. In Figs. 2(b) and 2(c) we show the significant effect of the applied deformation on the axial and tangential forces transmitted by the beams to the matrix. Initially, most of the load is carried by the two vertical beams in their axial direction. However, after the onset of instability the axial stress in the vertical beams is found to plateau, while both the axial and tangential force components transmitted by the horizontal beams significantly increase due to their stretching induced by the rotation of the metallic core. Hence, our results indicate that the applied deformation significantly alters the effective stiffness of the resonator.

Next, we numerically investigate the effect of such change in effective stiffness on the propagation of small amplitude elastic waves. For this, we calculate the dispersion relations for both the undeformed and deformed configurations using frequency domain analyses, fully accounting for the effect of precompression $[23,29]$.

In Fig. 3(a) we report the dispersion relation of the metamaterial in the undeformed configuration, while in Fig. 3(b) the Bloch mode shapes of the four lowest bands at the high-symmetry points, $X, M$, and $Y$ of the Brillouin zone are shown [29]. For clarity, in Fig. 3(a) we use red 


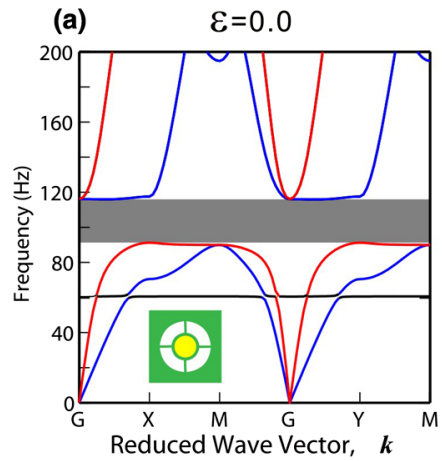

(b)

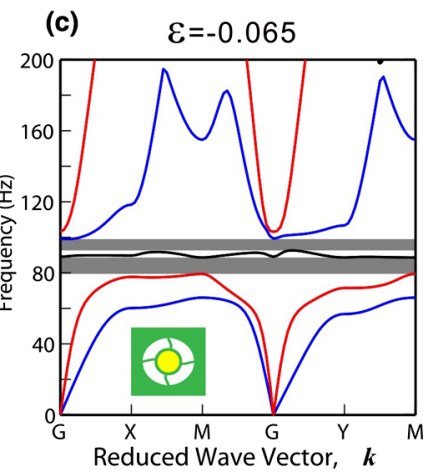

(d)

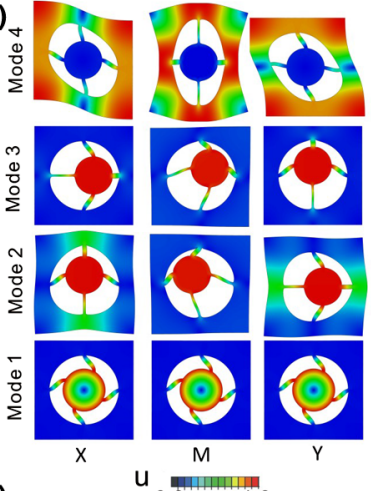

(d)

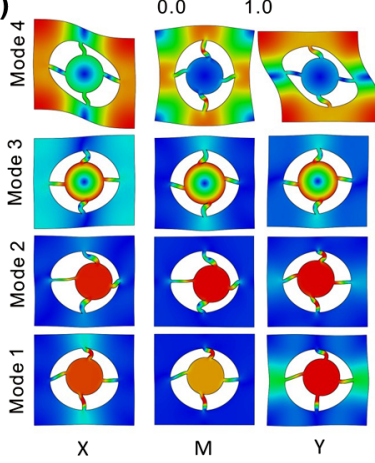

(e)

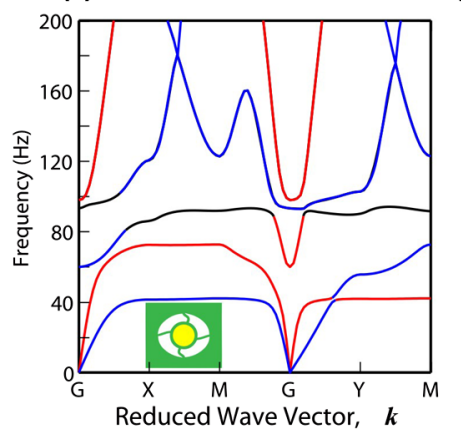

(f)

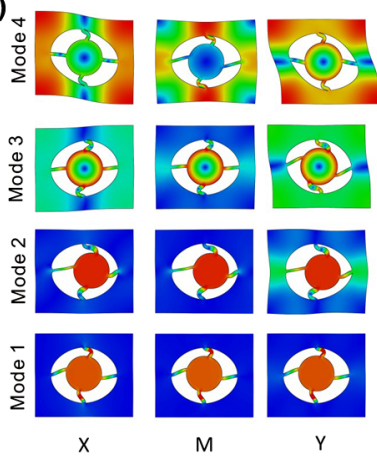

FIG. 3 (color online). Effect of the applied deformation on the band structure: Dispersion relations from Bloch-wave analysis for the infinite metamaterial in (a) the undeformed configuration and under uniaxial compressive strain (c) $\varepsilon=-0.065$ and (e) $\varepsilon=-0.10$. Shear dominated bands are colored in blue, pressure dominated bands in red and locally rotational bands in black. The grey region in (a) and (c) highlights the band gap induced by local resonance. The Bloch modes of the lowest four bands at highsymmetry points of the Brillouin zone $(X, M$, and $Y)$ [29] are shown in (b), (d), and (f) for $\varepsilon=0.0,-0.065$, and -0.10 , respectively. The distribution of the magnitude of the modal displacement field is shown.

and blue lines to represent pressure-dominated (longitudinal) and shear-dominated (transversal) wave modes, respectively. Interestingly, the first three modes at $X, M$, and $Y$ show a strong localization of vibration, with only the metallic core vibrating and the matrix mostly at rest. By contrast, the fourth mode at $X, M$, and $Y$ points has a global nature, since the entire matrix vibrates while the modal displacement of metallic core is negligible. We note that

similar modal patterns have been observed not only in acoustic metamaterials [15,34], but also in photonic crystals [35]. Further inspection of the modes reveals that the first localized mode [black line in Fig. 3(a)] is purely rotational, so that it has a very weak coupling with the propagating shear and pressure waves and does not open a band gap [34]. Differently, in the second and third modes the resonators interact with the shear and pressure waves, respectively. This coupling between the vibration of the metallic core and the propagating waves in the media generates a band gap, highlighted by the grey area in Figs. 3(a) and 3(c). Finally, we note that, although our numerical results indicate an anisotropy in the modes [see mode 2 in Fig. 3(b)], the locally resonant band gap is an omnidirectional complete gap.

The effect of deformation on the propagation of elastic waves is shown in Figs. 3(c) and 3(d) for $\varepsilon=-0.065$ and in Figs. 3(e) and 3(f) for $\varepsilon=-0.1$. Under small $\varepsilon$, the softening of the two vertical beams induced by buckling is found to dominate over the stiffening of the two horizontal beams induced by their stretching, so that the band gap frequency decreases, as shown in Fig. 3(c). However, due to the increase in tangential stiffness induced by instability, the rotational mode rises above the shear and pressure modes [see Fig. 3(d)] and splits the band gap. If we further increase the applied strain to $\varepsilon=-0.10$, the band gap then completely closes because of the modal transformation of the rotational band near the $G$ point [see Fig. 3(e)]. Therefore, our results clearly demonstrate that the proposed metamaterial acts as a reversible phononic switch, since the applied deformation can be exploited to tune and switch the band gap on or off.

To validate the numerical predictions, a sample of the locally resonant metamaterial comprising $6 \times 3$ unit cells (see Fig. 1) is fabricated using silicon rubber (Elite Double 32, Zhermack) and a mold-casting process with the mold prepared by $3 \mathrm{D}$ rapid prototyping. We first apply the desired level of strain $\varepsilon$ using a fixture made of acrylic plates and nylon bolts or nuts. Next, wave propagation in the sample is excited by an electrodynamic shaker (model K2025E013, Modal Shop), which is directly connected to one end of the sample to provide a white noise input signal over a broadband frequency range. The dynamic response at different levels of applied strain $\varepsilon$ is recorded using two miniature accelerometers (352C22, PCB Piezotronics) attached to both ends of the sample and the transmittance is computed as the ratio between the output and input acceleration signals (i.e., $\left\|A_{\text {out }}(\omega) / A_{\text {in }}(\omega)\right\|$ ).

In Fig. 4(a) we report the transmittance of the sample measured at $\varepsilon=0.0,-0.065$, and -0.10 . In the undeformed configuration (i.e., $\varepsilon=0.0$ ) a significant drop in the transmittance is observed between $80 \mathrm{~Hz}$ and $100 \mathrm{~Hz}$ with a pronounced minimum at $95 \mathrm{~Hz}$, which corresponds to the resonance frequency of vibration localization. Note 

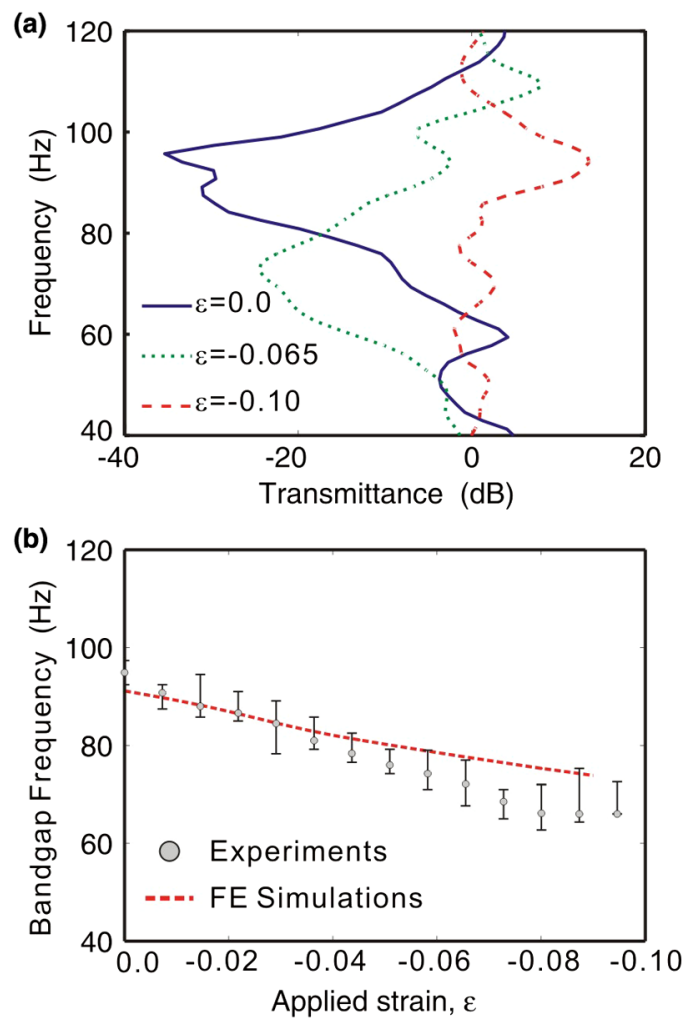

FIG. 4 (color online). Effect of the applied deformation on the band gap frequency: (a) Experimentally measured transmittance in a sample with $6 \times 3$ unit cells (see Fig. 1) at different levels of applied prestrains. (b) Evolution of the resonant band gap frequency as a function of the applied strain, $\varepsilon$. The markers and error bars represent the lowest transmittance frequency and the $\pm 3 \mathrm{~dB}$ band gap width measured experimentally. The dashed line indicates the lower edge of the band gap (i.e., frequency of resonance) predicted by the FE simulations for the corresponding infinite system.

that in finite-size locally resonant metamaterials the lowest transmittance is usually observed at the resonant frequency of the embedded resonators, which corresponds to the lower edge of the band gap predicted by the dispersion relation [15]. This result not only proves the existence of the band gap predicted by the simulations, but also quantitatively agrees with the FE results in which the lower edge of the band gap (i.e., the frequency with strongest resonance) was observed at $92 \mathrm{~Hz}$ [see Fig. 3(a)]. Moreover, as predicted by the numerical results, when a uniaxial strain $\varepsilon$ is applied, the experimentally measured band gap is found to shift towards lower frequencies. In particular, for $\varepsilon=-0.065$ the drop in the transmittance is shifted to the range of $60-85 \mathrm{~Hz}$, with the minimum at $76 \mathrm{~Hz}$. We also note that the band gap splitting predicted at $\varepsilon=-0.065$ by the FE simulations [see Fig 3(c)] is not observed in the measured transmittance. This discrepancy is attributed to finite-size effects such as boundary effects and nonuniform distribution of the predeformation, which are not fully accounted for in the simulations. Lastly, for $\varepsilon=-0.10$, the measured transmittance shows no band gap, confirming that the proposed metamaterial can be utilized as a phononic or acoustic switch.

The comparison between the resonant band gap frequency predicted by simulations and experiments as a function of the applied strain $\varepsilon$ is presented in Fig. 4(b). The markers and error bars represent the lowest transmittance frequency and the $\pm 3 \mathrm{~dB}$ band gap width measured experimentally, respectively. Moreover, the dashed line indicates the frequency of the lower edge of the band gap calculated numerically for the corresponding infinite system. Remarkably, we find excellent agreement between the two sets of data. Both experiments and simulations predict the band gap frequency first to decrease linearly as a function of $\varepsilon$ and then the band gap to completely disappear as $\varepsilon$ approaches -0.10 .

In summary, we demonstrated both numerically and experimentally that large deformation and local instability can be exploited to effectively control the response of locally resonant acoustic metamaterials. This remarkable behavior is achieved by introducing a structural coating comprising an array of elastic and highly deformable beams. Our results indicate that under externally applied load the stiffness of the beams varies significantly due to their buckling, altering the resonant frequency of the unit and providing a wide range of tunability for the band gap ( $\sim 30 \%$ in frequency). Furthermore, we showed that the proposed metamaterial can be utilized as an on or off acoustic switch, since a moderate level of applied uniaxial strain $(\varepsilon \sim-0.10)$ is enough to entirely suppress the band gap. Although in this Letter we only verified the concept with one specific set of material and geometric parameters, the response of the system is robust. Numerical parametric studies have also been performed by changing the arrangement of the resonating units, the number of beams in the structural coating, their slenderness, and the constitutive behavior of the matrix [29]. Our results indicate that the proposed mechanism works over a wide range of parameters, opening avenues for the design of smart systems that control the wave propagation depending on the applied deformation.

This work has been supported by Harvard MRSEC through Grant No. DMR-0820484 and by NSF through Grants No. CMMI-1120724 and No. CMMI-1149456 (CAREER). K. B. acknowledges start-up funds from the Harvard School of Engineering and Applied Sciences and the support of the Kavli Institute and Wyss Institute at Harvard University.

* Corresponding author. bertoldi@seas.harvard.edu

[1] M. Hussein, M. Leamy, and M. Ruzzene, Appl. Mech. Rev. 66, 040802 (2014). 
[2] A. Khelif, A. Choujaa, S. Benchabane, B. Djafari-Rouhani, and V. Laude, Appl. Phys. Lett. 84, 4400 (2004).

[3] M. Kafesaki, M. M. Sigalas, and N. Garcia, Phys. Rev. Lett. 85, 4044 (2000).

[4] A. Khelif, B. Djafari-Rouhani, J. O. Vasseur, P. A. Deymier, P. Lambin, and L. Dobrzynski, Phys. Rev. B 65, 174308 (2002).

[5] J.-H. Sun and T.-T. Wu, Phys. Rev. B 71, 174303 (2005).

[6] J.-H. Sun and T.-T. Wu, Phys. Rev. B 76, 104304 (2007).

[7] J. O. Vasseur, A. Hennion, B. Rouhani, F. Duval, B. Dubus, and Y. Pennec, J. Appl. Phys. 101, 114904 (2007).

[8] S. Cummer and D. Schurig, New J. Phys. 9, 45 (2007).

[9] D. Elser, U. L. Andersen, A. Korn, O. Glöckl, S. Lorenz, C. Marquardt, and G. Leuchs, Phys. Rev. Lett. 97, 133901 (2006).

[10] T. Elnady, A. Elsabbagh, W. Akl, O. Mohamady, V. M. Garcia-Chocano, D. Torrent, F. Cervera, and J. SánchezDehesa, Appl. Phys. Lett. 94, 134104 (2009).

[11] F. Casadei, L. Dozio, M. Ruzzene, and K. Cunefare, J. Sound Vib. 329, 3632 (2010).

[12] C. Kittel, Am. J. Phys. 35, 547 (1967).

[13] D. R. Smith, W. J. Padilla, D. C. Vier, S. C. Nemat-Nasser, and S. Schultz, Phys. Rev. Lett. 84, 4184 (2000).

[14] P. Gay-Balmaz and O. J. F. Martin, J. Appl. Phys. 92, 2929 (2002).

[15] Z. Liu, X. Zhang, Y. Mao, Y. Zhu, Z. Yang, C. Chan, and P. Sheng, Science 289, 1734 (2000).

[16] L. Airoldi and M. Ruzzene, New J. Phys. 13, 113010 (2011).

[17] F. Casadei, B. Beck, K. A. Cunefare, and M. Ruzzene, Int. J. Solids Struct. 23, 1169 (2012).

[18] D. Bigoni, S. Guenneau, A. B. Movchan, and M. Brun, Phys. Rev. B 87, 174303 (2013).

[19] N. Fang, D. Xi, J. Xu, M. Ambati, W. Srituravanich, C. Sun, and X. Zhang, Nat. Mater. 5, 452 (2006).
[20] Y. Lai, Y. Wu, P. Sheng, and Z. Zhang, Nat. Mater. 10, 620 (2011).

[21] B. L. Davis and M. I. Hussein, Phys. Rev. Lett. 112, 055505 (2014).

[22] K. Bertoldi and M. C. Boyce, Phys. Rev. B 77, 052105 (2008).

[23] P. Wang, J. Shim, and K. Bertoldi, Phys. Rev. B 88, 014304 (2013).

[24] S. Rudykh and M. C. Boyce, Phys. Rev. Lett. 112, 034301 (2014).

[25] F. Casadei, T. Delpero, A. Bergamini, P. Ermanni, and M. Ruzzene, J. Appl. Phys. 112, 064902 (2012).

[26] A. Bergamini, T. Delpero, L. De Simoni, L. Di Lillo, M. Ruzzene, and P. Ermanni, Adv. Mater. 26, 1472 (2014).

[27] F. Casadei and K. Bertoldi, J. Appl. Phys. 115, 034907 (2014).

[28] Z. Liu, C. T. Chan, and P. Sheng, Phys. Rev. B 65, 165116 (2002).

[29] See Supplemental Material at http://link.aps.org/ supplemental/10.1103/PhysRevLett.113.014301 for details of numerical procedures and additional parametric studies, which includes Refs. [24,30-33].

[30] L. Brillouin, Wave Propagation in Periodic Structures (McGraw-Hill, New York, 1946).

[31] M. Maldovan and E. Thomas, Periodic Materials and Interference Lithography for Photonics, Phononics and Mechanics (Wiley-VCH, Weinheim, 2009).

[32] A. Gent, Rubber Chem. Technol. 69, 59 (1996).

[33] L. Treloar, Trans. Faraday Soc. 40, 59 (1944).

[34] G. Wang, X. Wen, J. Wen, L. Shao, and Y. Liu, Phys. Rev. Lett. 93, 154302 (2004).

[35] J. D. Joannopoulos, S. G. Johnson, J. N. Winn, and R. D. Meade, Photonic Crystals: Molding the Flow of Light (Princeton University Press, Princeton, NJ, 2008), 2nd ed., p. 70. 


\title{
Supporting Information for \\ Harnessing buckling to design tunable locally resonant acoustic metamaterials
}

\author{
Pai Wang, ${ }^{1}$ Filippo Casadei, ${ }^{1}$ Sicong Shan, ${ }^{1}$ and Katia Bertoldi ${ }^{1,2, *}$ \\ ${ }^{1}$ School of Engineering and Applied Sciences, Harvard University, Cambridge, Massachusetts 02138, USA \\ ${ }^{2}$ Kavli Institute, Harvard University, Cambridge, Massachusetts 02138, USA
}

\section{CALCULATION OF DISPERSION RELATIONS}

Periodic Structure and Irreducible Brillouin Zone

The propagation of elastic waves within the acoustic metamaterial is investigated numerically by considering a $2 \mathrm{D}$ periodic solid of infinite extent characterized by a primitive cell (i.e. minimum unit identified in the periodic structure) spanned by the lattice vectors $\mathbf{a}_{1}$ and $\mathbf{a}_{2}$, as shown in Fig. S1A for the undeformed configuration. Thus, any spatial function field, $\phi(\mathbf{x})$, in the infinite periodic structure satisfies the condition:

$$
\phi(\mathbf{x}+\mathbf{p})=\phi(\mathbf{x})
$$

where

$$
\mathbf{p}=p_{1} \mathbf{a}_{1}+p_{2} \mathbf{a}_{2}
$$

where $p_{1}$ and $p_{2}$ are arbitrary integers. Note that when the periodic solid is deformed the lattice vectors $\mathbf{a}_{1}$ and $\mathbf{a}_{2}$ change, as indicated in Fig. S1C, for an uni-axially deformed configuration.

A

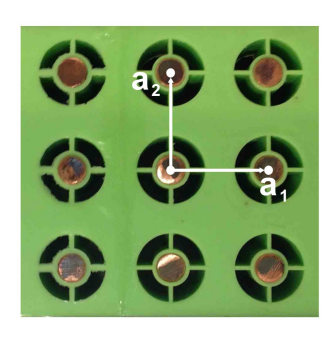

C

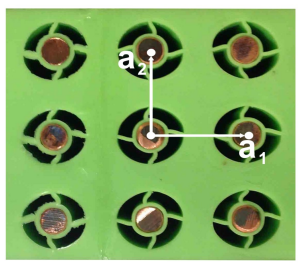

B $\quad b_{2}$
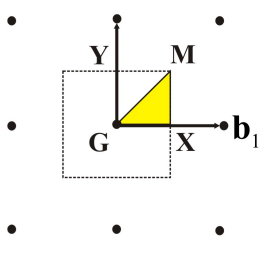

$\mathbf{b}_{2}$

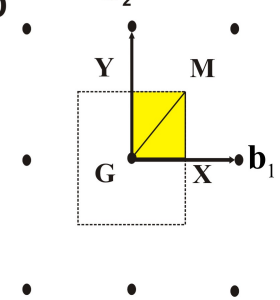

Figure S1: A) Crystal lattice in the undeformed configuration. B) Reciprocal lattice in the undeformed configuration. C) Crystal lattice in an uni-axially deformed configuration. D) Reciprocal lattice in an uni-axially deformed configuration.

For later use, we define the reciprocal lattice specified by the reciprocal lattice vectors $\mathbf{b}_{1}$ and $\mathbf{b}_{2}$ defined such that

$$
\mathbf{a}_{i} \cdot \mathbf{b}_{j}=2 \pi \delta_{i j}
$$

where $\delta_{i j}$ is the Kronecker delta. It follows that $\mathbf{b}_{1}$ and $\mathbf{b}_{2}$ are defined as

$$
\mathbf{b}_{1}=2 \pi \frac{\mathbf{a}_{2} \times \mathbf{z}}{\|\mathbf{z}\|^{2}}, \quad \mathbf{b}_{2}=2 \pi \frac{\mathbf{z} \times \mathbf{a}_{1}}{\|\mathbf{z}\|^{2}}
$$


where $\mathbf{z}=\mathbf{a}_{1} \times \mathbf{a}_{2}$.

As shown in Figs. S1B and S1D, the reciprocal lattice is characterized by spatial periodicity $\mathbf{g}$

$$
\mathbf{g}=g_{1} \mathbf{b}_{1}+g_{2} \mathbf{b}_{2}
$$

with $g_{1}$ and $g_{2}$ being arbitrary integers.

\section{Frequency-domain analyses}

In order to obtain the dispersion relation of the propagating waves in the periodic media at different levels of deformation, frequency domain analyses are performed on the deformed configurations generated by the post-buckling analysis. Bloch-type boundary conditions are applied:

$$
\mathbf{u}(\mathbf{x}+\mathbf{r})=\mathbf{u}(\mathbf{x}) e^{i \mathbf{k} \cdot \mathbf{r}}
$$

where $\mathbf{k}$ and $\mathbf{r}$ denote the bloch-wave vector and spatial periodicity in the deformed configuration, respectively.

Focusing on the propagation of small-amplitude waves, we solve the linearized wave equation on the non-linearly obtained post-buckling base state by using a perturbation method to obtain the dispersion relations $\omega=\omega(\mathbf{k})$. Details of this formulation and implementation are given in a previous publication [1].

Note that for a 2D infinite periodic structure with spatial periodicity defined by (S1) and (S2), the periodicity in the reciprocal $\mathbf{k}$-space of the dispersion relation is given by [2]:

$$
\omega(\mathbf{k}+\mathbf{g})=\omega(\mathbf{k})
$$

with $\mathbf{g}$ defined in (S5). Hence, due to translational symmetry specified by (S7), we only need to study $\omega(\mathbf{k})$ for $\mathbf{k}$ vectors in the first Brillouin zone [2], indicated by the area inside the square GXMY in Fig. S1B and the area inside the rectangle GXMY in Fig. S1D. Moreover, the domain can be further reduced by taking advantage of rotational, reflectional and inversional symmetries of the first Brillouin zone. This allows us to define the irreducible Brillouin zone (IBZ) [3], shown as the yellow triangle GXM in Fig. S1B and yellow rectangle GXMY in Fig. S1D).

The phononic bandgaps are identified by checking all eigen-frequencies $\omega(\mathbf{k})$ for $\mathbf{k}$ vectors on the perimeter of the IBZ. The bandgaps (i.e. range in frequencies for which the propagation of waves is barred) are given by the frequency ranges within which no $\omega(\mathbf{k})$ exist. Numerically, a discrete set of $\mathbf{k}$ vectors on the IBZ perimeter needs to be chosen in the band-gap calculation. For the simulations presented in this paper, twenty uniformly-spaced points on each edge of the IBZ are used for the purpose of identifying band-gaps.

\section{PARAMETRIC STUDY}

In the manuscript we present results only for one specific configuration that comprises a square array of locally resonant units consisting of metallic circular cores with radius $R_{0}=7.9 \mathrm{~mm}$ connected to the matrix by four beams with length $L_{0}=16.9 \mathrm{~mm}$ and thickness $t_{0}=1.8 \mathrm{~mm}$.

Although in the main text we report the results obtained using only this specific set of material and geometric parameters, the robustness of the proposed concept has been verified through several parametric studies. In this section, we present the results of the parametric studies we conducted to investigate the effects of: $(i)$ Arrangement of the resonating units; ( $i i$ ) Number of elastic beams in the structural coating; (iii) Slenderness of the beams; and

(iv) Constitutive behavior of the matrix material. 
Effect of the arrangement of the resonating units

Although in the main text we report results only for an acoustic metamaterial comprising a square array of resonating units, the response of the system is not affected by the arrangement of the mechanical resonators within the matrix. In fact, the coupling effects between different resonators are negligible as long as they are well-separated by the matrix material. To demonstrate this important point, we have calculated the dispersion relation for a system with resonators arranged also in triangular and hexagonal arrays, keeping the density of resonators per unit area constant in all cases. The results are reported in Fig. S2 and clearly indicate that the bandgap of the system is not affected by the arrangement of the resonators.
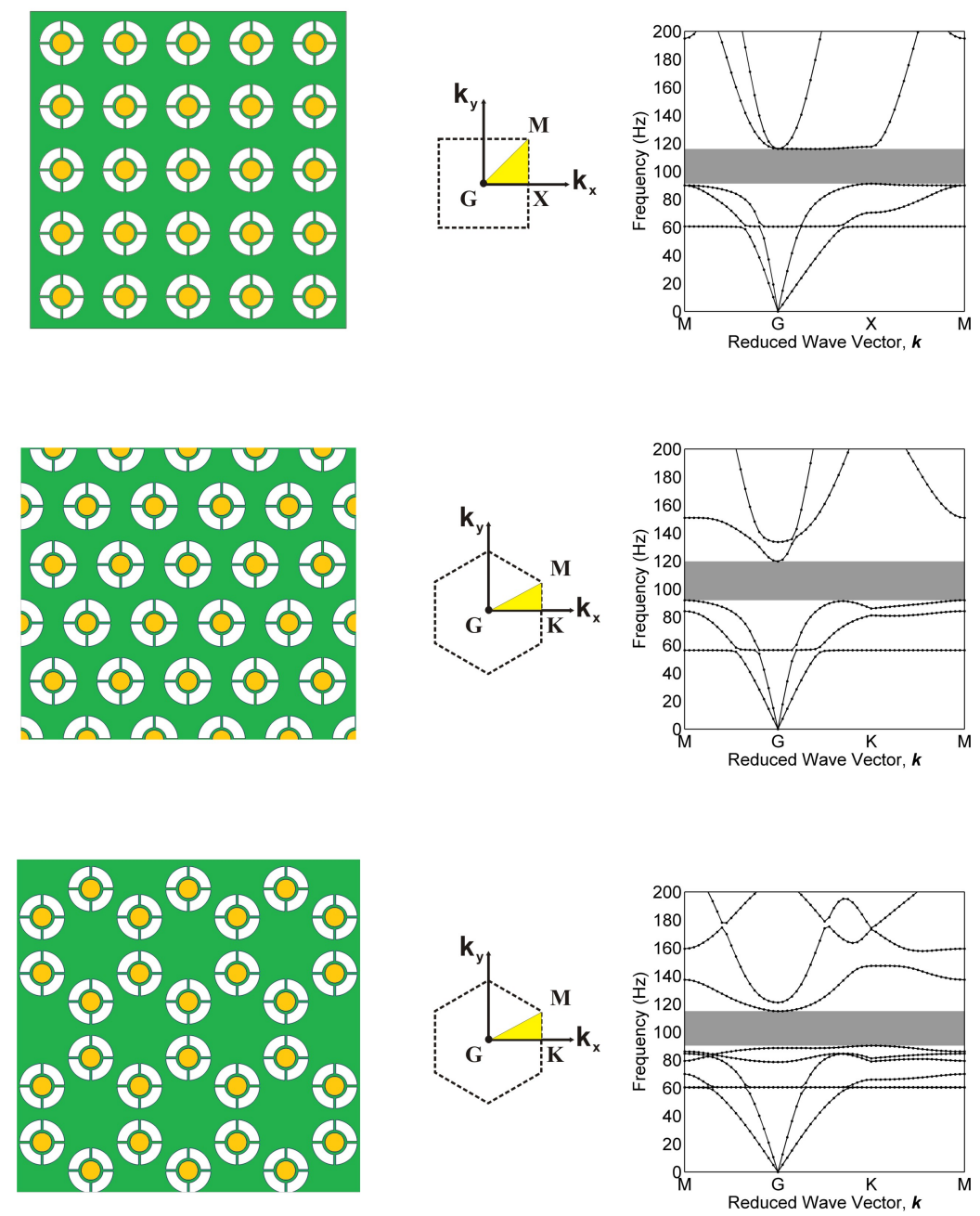

Figure S2: Dispersion relations calculated for a metamaterials comprising a square (top), triangular (center) and hexagonal (bottom) array of resonating units. The dispersion relations are calculated in the undeformed configuration. 
Although in the main text we only present results for a configuration with a structural coating that comprises four beams, the proposed mechanism also works for different number of beams. To highlight this point, in Fig. S3 we report the dispersion relation calculated in the undeformed configuration for resonant units comprising $3,4,5$ and 6 beams (with length $L_{0}=16.9 \mathrm{~mm}$ and thickness $t_{0}=1.8 \mathrm{~mm}$ ). Clearly, in all four configurations a locally resonant bandgap is generated, as highlighted by the grey region. We also note that the frequency of such a bandgap increases with the number of beams. This is due to the fact that more beams make the structural coating effectively stiffer, resulting in a higher resonant frequency for the resonating unit.
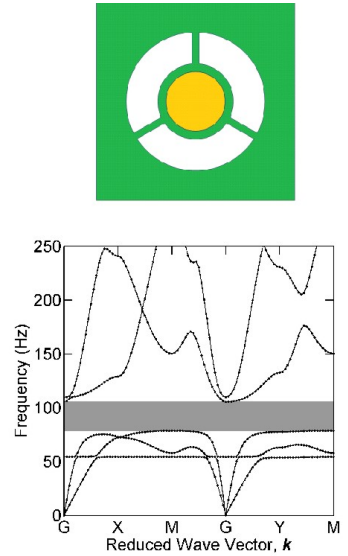
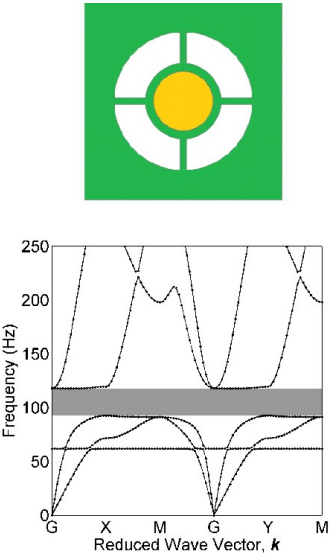
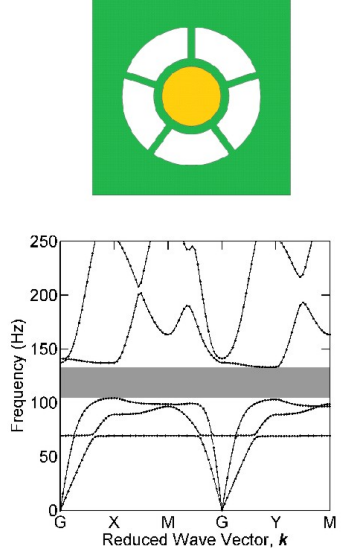
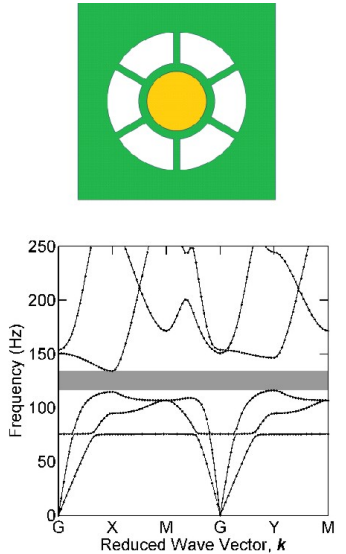

Figure S3: Dispersion relations calculated for resonant units comprising 3, 4, 5 and 6 beams (with length $L_{0}=16.9 \mathrm{~mm}$ and thickness $t_{0}=1.8 \mathrm{~mm}$ ). The dispersion relations are calculated in the undeformed configuration.

In addition, our numerical results also indicate that in all the configurations reported in Fig. S3 the applied deformation can be exploited as an effective mechanism to control the propagation of elastic waves. As an example, in Fig. S4 we report results for the 3-beam design under applied uni-axial loading in the vertical direction up to $\epsilon=-0.10$. On the top part of the figure, snapshots of the unit cell at different levels of deformation $(\varepsilon=0.0,-0.065,-0.10)$ are shown, indicating that in this case the applied compression also leads to buckling of the beams. Moreover, on the bottom part of the figure we report the dispersion relation for $\varepsilon=0.0,-0.065$ and -0.10 . These results clearly indicate that for this design the applied deformation can also be exploited to switch the bandgap on and off, confirming the robustness of the proposed mechanism.

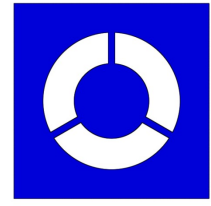

$\varepsilon=0.0$

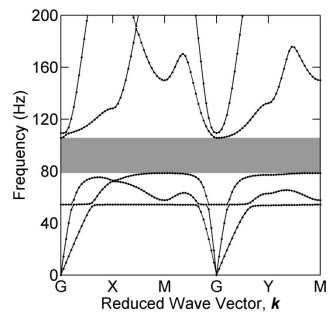

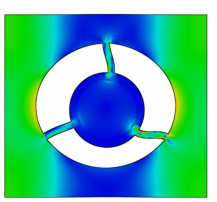

$\varepsilon=-0.065$

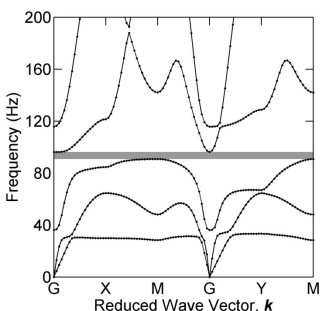

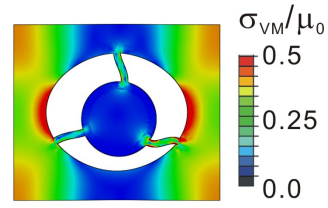

$\varepsilon=-0.10$

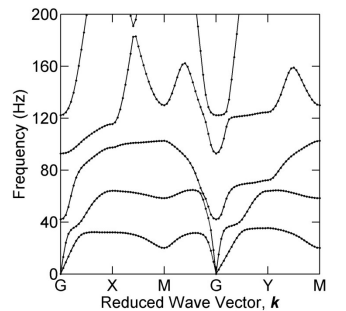

Figure S4: Effect of the applied deformation on the static and dynamic response of a resonating unit comprising 3 beams. 
Although in the main text we present results for a metamaterial whose structural coating comprises an array of beams with aspect ratio $t_{0} / L_{0}=1.8 / 16.9$, we also investigated the effect of the slenderness of the beams on the dynamic response of the system. In Fig. S5 we report the evolution of the resonant bandgap frequency predicted by simulations for a resonant unit comprising 4 beams with $t_{0} / L_{0}=2.5 / 16.9,2.0 / 16.9,1.8 / 16.9$ (original design parameter) and 1.5/16.9. Since an increase of the beam's aspect ratio $t_{0} / L_{0}$ results in higher effective stiffness of the structural coating, the bandgap frequency is found to monotonically increase as a function of $t_{0} / L_{0}$. However, in all the cases the bandgap frequency is found to first monotonically decrease as a function of the applied strain $\varepsilon$. The bandgap then completely closes at $\varepsilon \sim-0.10$.

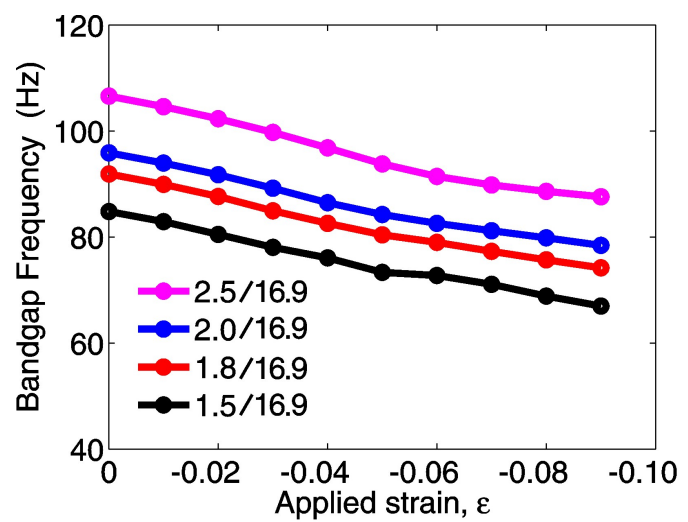

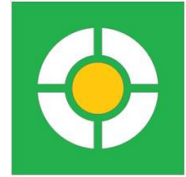

$2.5 / 16.9$

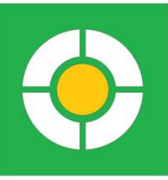

$1.8 / 16.9$

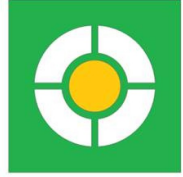

$2.0 / 16.9$

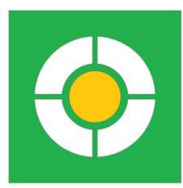

$1.5 / 16.9$

Figure S5: Effect of the slenderness of the elastic beams on the bandgap frequency.

Finally, we highlight the fact that to generate a locally resonant bandgap a large contrast in stiffness between the matrix and the structural coating is required. Therefore, for very thick beams the locally resonant bandgap is completely suppressed, since the effective stiffness of the coating is comparable to that of the matrix when the beam thickness is increased above a certain limit. The suppression of the bandgap in the undeformed configuration can be clearly seen in Fig. S6, where we report the dispersion relation for a coating comprising beams with $t_{0} / L_{0}=5 / 16.9$.

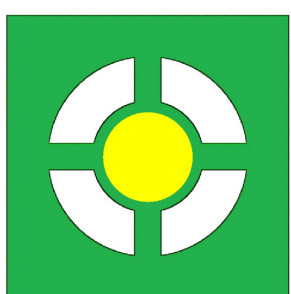

Beam Slenderness $5.0 / 16.9$

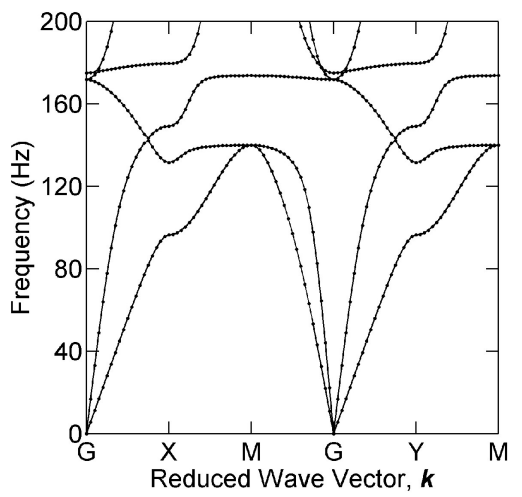

Figure S6: For very thick beams $\left(t_{0} / L_{0}=5 / 16.9\right)$ the locally resonant bandgap is suppressed, since the effective stiffness of the coating is comparable to that of the matrix. 
Effect of the constitutive behavior of the matrix material

In the main text we report results for a metamaterial in which both the beams and the matrix are made of an elastomeric material whose response is described by a nearly incompressible Neo-Hookean model with initial shear modulus $\mu_{0}$ and Poisson's ratio $\nu_{0}=0.499$. The response of such material is weakly nonlinear and the stiffening effect induced by the applied deformation is negligible in this case. However, we also numerically investigated the effect of material nonlinearities on the propagation of elastic waves in the metamaterial using the Gent model [4].

The nearly-incompressible version of the strain energy function proposed by Gent [4] is given by

$$
\begin{aligned}
W\left(I_{1}, J\right)= & -\frac{\mu_{0}}{2} J_{m} \log \left(1-\frac{I_{1}-3}{J_{m}}\right)-\mu_{0} \log (J) \\
& +\left(\frac{K_{0}}{2}-\frac{\mu_{0}}{J_{m}}\right)(J-1)^{2}
\end{aligned}
$$

where $\mu_{0}$ and $K_{0}$ are the initial shear and bulk moduli. Here, $J_{m}$ denotes a material constant related to the strain at saturation, since the stress becomes infinite as $J_{m}-I_{1}+3$ approaches zero. Moreover, for a given deformation gradient tensor $\mathbf{F}, I_{1}=\operatorname{trace}\left(\mathbf{F}^{T} \mathbf{F}\right)$ and $J=\operatorname{det}(\mathbf{F})$. We note that as $J_{m} \rightarrow+\infty$ the Gent model reduces to the Neo-Hookean model [5], for which the nearly-incompressible version of the strain energy density function is given by

$$
W\left(I_{1}, J\right)=\frac{\mu_{0}}{2}\left(I_{1}-3\right)-\mu_{0} \log (J)+\frac{K_{0}}{2}(J-1)^{2}
$$

In our numerical study we use both the Gent (with $J_{m}=2.0$ ) and Neo-Hookean (equivalent to Gent model with $\left.J_{m}=+\infty\right)$ models to capture the response of the matrix and the beams. The results are summarized in Fig. S7 and clearly indicate that the saturation strain of the constituent material (represented by $J_{m}$ ) has no significant influence on the bandgap evolution of the metamaterial as a function of the applied deformation.

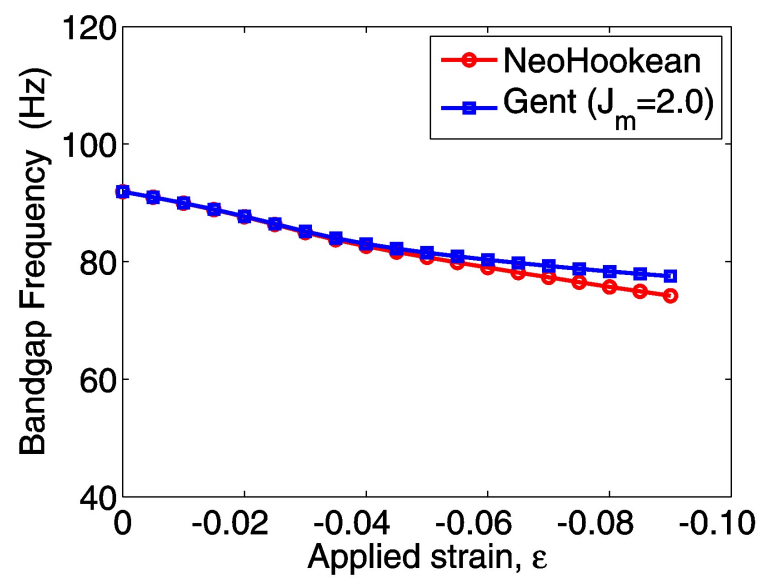

Figure S7: The saturation strain of the constituent material (represented by $J_{m}$ ) has no significant influence on the bandgap evolution of the metamaterial as a function of the applied deformation.

* Corresponding author. bertoldi@seas.harvard.edu

[1] P. Wang, J. Shim, and K. Bertoldi, Phys. Rev. B 88, 014304 (2013).

[2] L. Brillouin, Wave Propagation in Periodic Structures (McGraw-Hill, 1946).

[3] M. Maldovan and E. Thomas, Periodic Materials and Interference Lithography for Photonics, Phononics and Mechanics (Wiley-VCH, 2009).

[4] A. Gent, Rubber Chem. Tech. 69, 59 (1996).

[5] L. Treloar, Trans. Faraday Soc. 40, 59 (1944). 